\title{
Uma ferramenta de Visualização da Informação para analisar o comportamento do aluno em um ambiente e-learning e sua trajetória de aprendizagem
}

\author{
An Information Visualization tool to analyze student's behavior \\ in an e-learning environment and his learning path
}

Visualização da Informação, Trajetória de Aprendizagem, e-learning.

\begin{abstract}
A visualização é o processo de apresentar os resultados de um dado processo de extração ou de uma determinada busca, de forma a permitir que o usuário tenha acesso ao maior número possível de dados resultantes de uma pesquisa, de forma usável e clara. Este trabalho tem como objetivo apresentar uma ferramenta de visualização da informação para analisar o comportamento do aluno em um ambiente e-learning e explorar o conceito de trajetória de aprendizagem para ser incorporado futuramente na ferramenta. O objetivo da ferramenta é possibilitar que o professor compreenda melhor o comportamento de seus alunos frente ao ambiente e dê suporte à tomada de decisões em relação ao conteúdo pedagógico adaptado às necessidades dos alunos. Como resultado, a ferramenta foi implementada e está em uso no ambiente AdaptWeb ${ }^{\circledR}$ (Ambiente de Ensino-Aprendizagem Adaptativo na Web).
\end{abstract}

Visualization is the process of presenting the results of a given extraction process or a particular search, in order to allow the user to have access to the greatest number of data resulting from a research, in a usable and clearly way. This paper aims to present an information visualization tool for analyzing student's behavior in an e-learning environment and explore the concept of learning path to be incorporated in the future. The purpose of this tool is to allow the teacher to better understand his students' behavior in front of the environment and support the decision making related to the educational content adapted to students' needs. As a result, the tool has been implemented and is in use in AdaptWeb $®$ environment (Adaptive Web-Based Learning Environment).

\section{Introdução}

A visualização é o processo de apresentar os resultados de um dado processo de extração ou de uma determinada busca, de forma a permitir que o usuário tenha acesso ao maior número possível 
de dados resultantes de uma pesquisa, de forma usável e clara. A visualização da informação (InfoVis) é uma área que tem como objetivo auxiliar a análise de um grande conjunto de dados através de representações gráficas manipuláveis. Desta forma, através das técnicas de InfoVis um grande conjunto de dados pode ser representado graficamente de forma a facilitar sua análise. Além de representar estes dados de forma visual, as técnicas de InfoVis permitem que o usuário manipule os dados representados e a representação destes para encontrar as informações que procura.

Esse processo visual de análise e interpretação dos resultados é ainda mais importante quando tratamos de ambientes virtuais de aprendizagem (AVA), também denominados ambientes e-learning. Os ambientes e-learning podem se beneficiar do uso de técnicas de visualização da informação. Em ambientes e-learning, uma das dificuldades enfrentadas pelos professores é conhecer e entender os alunos de forma a adaptarem os materiais didáticos de acordo com suas necessidades. Além disso, o professor também pode desejar verificar a assiduidade dos alunos, seus tópicos de interesse, seus estilos e preferências navegacionais e outros fatores que podem influenciar no modo que o professor prepara sua disciplina. $\mathrm{O}$ ambiente e-learning adaptativo AdaptWeb ${ }^{\circledR}$ (Ambiente de EnsinoAprendizagem Adaptativo na Web) coleta dados navegacionais dos alunos e calcula métricas para que as informações e os padrões de comportamento dos alunos possam ser apresentados aos professores e auxiliá-los nesta tarefa.

Este artigo apresenta uma ferramenta de visualização da informação desenvolvida através do uso de técnicas de representação e técnicas de interação, e implementada no ambiente AdaptWeb ${ }^{\circledR}$ para auxiliar o professor a entender o comportamento do aluno em suas disciplinas; este artigo também destaca o conceito de trajetória de aprendizagem, buscando como trabalhos futuros incorporá-la na ferramenta de visualização.

Este artigo está estruturado como segue. A seção 2 apresenta o conceito de visualização da informação, suas características, modelo de referências, técnicas de representação e técnicas de interação. A seção 3 explora o conceito de trajetória de aprendizagem. A seção 4 apresenta a ferramenta desenvolvida, mostrando o ambiente e-learning na qual foi baseada; as etapas de análise, representação e interação com os resultados; e trabalhos futuros. A seção 5 apresenta as considerações finais do trabalho. Por fim, na Seção 6 os agradecimentos são apresentados, seguidos das referências.

\section{Visualização da Informação}

Para transformar os dados em informação com significado, que auxiliem em processos de decisão e que gerem conhecimento, uma opção é criar representações visuais, pois estas tornam possível a 
exploração, a análise e a compreensão humana (sTASkO, 2008). Este é o objetivo da Visualização da Informação (InfoVis), uma área que combina aspectos de computação gráfica, interação humanocomputador (IHC) e mineração de dados para representar grandes conjunto de dados através de representações gráficas manipuláveis que possibilitam que o usuário interaja com dados de maneira rápida e eficiente e descubra características, padrões e tendências, i.e., transforme dados em informações significativas (GHERSON, EICK \& CARD, 1998).

Para criar as representações visuais, pode-se utilizar um modelo de referência visto que este permite que sejam identificados os componentes essenciais para o uso ou desenvolvimento de uma técnica (FREITAS ET AL. 2001) e também auxilia na compreensão do processo de transformação dos dados em representações visuais. Alguns modelos de referência estão presentes na literatura, tais como os de Haber e McNabb (1990); Campo, Orosko e Teyseyre (1997); Chi e Riedl (1998); e Card, Mackinlay e Shneiderman (1999). O processo de transformação dos dados em representações visuais é similar em todos estes modelos, no entanto o modelo de Card, Mackinlay e Shneiderman (1999), ilustrado na Figura 1, é o único que considera os impactos que a interação do usuário pode causar ao interagir com a visualização.

No processo descrito por Card, Mackinlay e Shneiderman (1999), os dados brutos são transformados em uma tabela de dados, composta de variáveis (colunas), itens (linhas) e metadados que descrevem cada uma das variáveis. Em seguida, os dados contidos na tabela (dados transformados) são mapeados para a estrutura visual escolhida para representá-los. Após realizar este mapeamento a estrutura visual resultante é exibida para o usuário que poderá analisar os resultados de forma interativa.

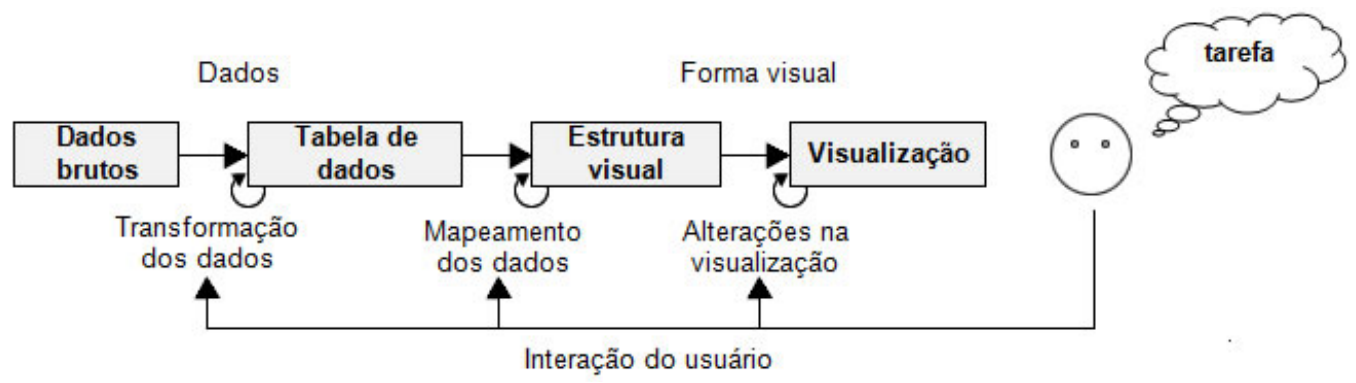

Figura 1 Modelo de InfoVis

Fonte: Tradução de Card, Mackinlay e Shneiderman, 1999.

A análise interativa dos dados permite que o usuário realize alteração nos dados e na estrutura de visualização, podendo exigir que o sistema realize novamente a transformação de dados brutos para uma tabela de dados; o mapeamento dos dados transformados em uma estrutura visual, que pode ser a mesma estrutura ou uma nova; e a exibição da estrutura visual. De acordo com a ação do usuário, o 
sistema pode retornar a qualquer uma das etapas. Por exemplo, se o usuário realiza um filtro por período nos dados, isto força que o sistema retorne a primeira etapa: a transformação dos dados brutos na tabela de dados. Por outro lado, se o usuário alterar a estrutura de visualização, o sistema retorna a etapa do mapeamento visual e mapeia novamente os dados transformados para a estrutura visual escolhida.

Durante este processo, obrigatoriamente são empregadas as técnicas de representação e as técnicas de interação. As técnicas de representação correspondem aos gráficos ou figuras utilizadas para representar os dados. De acordo com Card, Mackinlay e Shneiderman (1999) estas técnicas podem ser classificadas em quatro categorias:

- Ortogonais: São técnicas que utilizam no máximo três eixos e algum tipo de marcação para representar os dados. Este tipo de técnica é bastante efetivo para representar dados tabulares com poucas variáveis. Alguns exemplos desta categoria são gráficos de barras, gráficos de setores, gráficos de bolas, gráficos de dispersão, gráficos de superfície e mapas geográficos.

- Multidimensionais: Estas técnicas são utilizadas quando os dados tabulares possuem mais de três variáveis e as visualizações ortogonais são insuficientes. O gráfico de coordenadas paralelas e o gráfico de radar são exemplos de técnicas desta categoria.

- Redes: As redes representam relacionamentos entre os elementos, e.g., rede de computadores e modelos entidaderelacionamento (modelo de representação da estrutura de um banco de dados). Uma rede pode ser representada por um grafo (direcional ou não), composto de vértices que representam os relacionamentos e por arestas que representam o relacionamento entre estes elementos; e também por matrizes (VAZ \& CARVALHO, 2004).

- Árvores: Assim como as redes, as árvores representam relações. No entanto, no caso das árvores, estas relações são de hierarquia ou composição entre elementos, e.g., diretório de arquivos e árvores genealógicas (vAZ \& CARVALHO, 2004). De acordo com Graham e Kennedy (2010) há cinco maneiras de representar árvores: grafos (nós e arestas), aninhamentos, adjacências, identações e matrizes. Alguns exemplos de técnicas para representar árvores são as treemaps e árvores hiperbólicas.

As técnicas de interação correspondem aos mecanismos disponíveis no sistema para que o usuário manipule as informações apresentadas, propiciando as condições necessárias para a compreensão de grandes conjuntos de dados (FREITAS ET AL., 2001). Yi et al. (2007) classificam as técnicas de interação em sete categorias: 
- Seleção: Permitem que o usuário selecione os itens de seu interesse, assim quando muitos itens são representados e as representações são alteradas o usuário pode facilmente acompanhar estes itens e distingui-lo dos demais.

- Exploração: Permitem que o usuário examine diferentes subconjuntos de dados, mostrando novos itens e escondendo outros.

- Reconfiguração: Fornecem ao usuário diferentes perspectivas dos dados representados através de alterações na organização dos itens (e.g., alinhamento, ordem).

- Codificação: Permitem que o usuário altere a representação visual dos dados (e.g., cor, tamanho, forma, estrutura visual).

- Abstração/elaboração: Habilitam o usuário a ajustar o nível de abstração dos dados representados, i.e., trocar de uma visão geral para uma visão detalhada e vice-versa.

- Filtragem: Permitem que o usuário altere o conjunto de dados representado com base em condições específicas (e.g., mostrar as vendas referentes ao mês de dezembro, mostrar as vendas referentes à seção Informática).

- Conexão: Permitem que o usuário destaque associações e relações entre itens representados ou mostram itens escondidos relevantes a outro item.

Conforme descrito acima, a visualização é o processo de apresentar os resultados de um dado processo de extração ou de uma determinada busca, de modo que o usuário possa analisar um grande conjunto de dados de forma usável e clara. Esse processo visual de análise e interpretação dos resultados é muito importante quanto tratamos de ambientes e-learning, visto que os professores podem analisar o comportamento do aluno em um ambiente e-learning (e.g., verificar a assiduidade dos alunos, seus tópicos de interesse, seus estilos e preferências navegacionais) e sua trajetória de aprendizagem. Nas próximas seções são explorados os aspectos relacionados a ambientes e-learning.

\section{Trajetória de aprendizagem}

A trajetória de aprendizagem está relacionada ao caminho que o aluno percorre dentro de uma determinada disciplina em um ambiente de aprendizagem (schoonemboom ET AL., 2007). O "caminho é o conjunto de passos dado pelo usuário ao interagir com o ambiente" (PALAZZO ET AL. 2014), é o conjunto de páginas que o usuário acessou, em que ordem e com que frequência o fez. Identificar a trajetória de aprendizagem de um aluno auxilia na identificação do padrão de comportamento e do modelo de usuário, podendo ser utilizado para adaptar a interface principalmente no que se refere à navegação 
(PAlAZzo ET AL., 2014). Além disso, visualizar a trajetória do aluno na disciplina pode auxiliar o professor a entender o processo de aprendizagem de cada aluno, ou de uma turma inteira, e identificar possíveis dificuldades encontradas ao desenvolver determinadas atividades. Na literatura, há algumas ferramentas que permitem a análise da trajetória de aprendizagem do aluno.

A ferramenta LeMo (FORTENBACHER ET AL., 2013) foi desenvolvida para capturar, entre outras características, a trajetória de um aluno em um AVA. Essa ferramenta possui compatibilidade com diferentes AVAs, tais como o Moodle, Clix e Chemgapedia, cada um com suas próprias características. Após realizar as análises, o sistema gera diferentes formas de visualização, e.g, grafo de páginas acessadas (próprio caminho), caminhos frequentes, tempo das atividades, objetos mais acessados, etc. Na Figura 2 pode-se ver diferentes visualizações criadas pela LeMo.

Outra ferramenta que também está relacionada com a captura do caminho percorrido pelo usuário é a WET (PASCUAL-CID, 2008), que pode ser utilizada em diversos websites, inclusive em AVAs. Esta ferramenta possui como principal característica a capacidade de criar uma representação visual baseando-se na estrutura, no conteúdo e na utilização do website analisado. As três principais visualizações que essa ferramenta nos permite fazer são: representação hierárquica da estrutura do website, caminhos percorridos mais comuns a partir de uma determinada página de entrada e um grafo ilustrando todos os caminhos percorridos. Na Figura 3 é possível observar uma representação de uma trajetória gerada utilizando a WET.

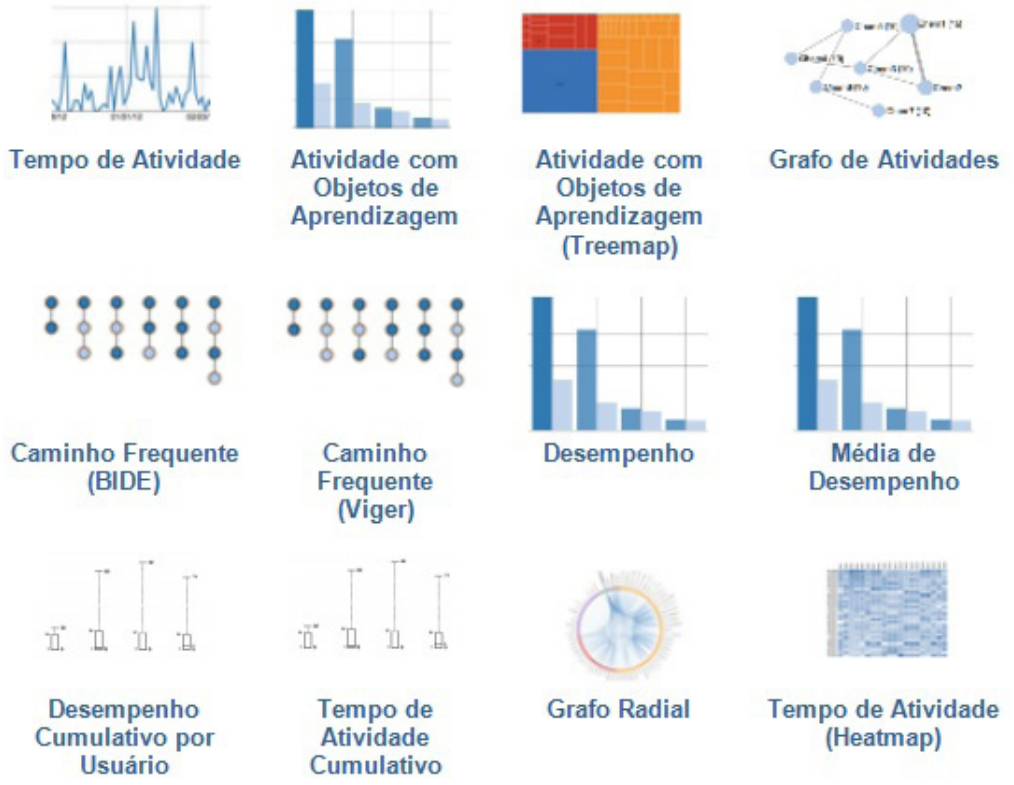

Figura 2 Diferentes visualizações na ferramenta LeMo Fonte: Tradução de FORTENBACHER ET AL., 2013. 


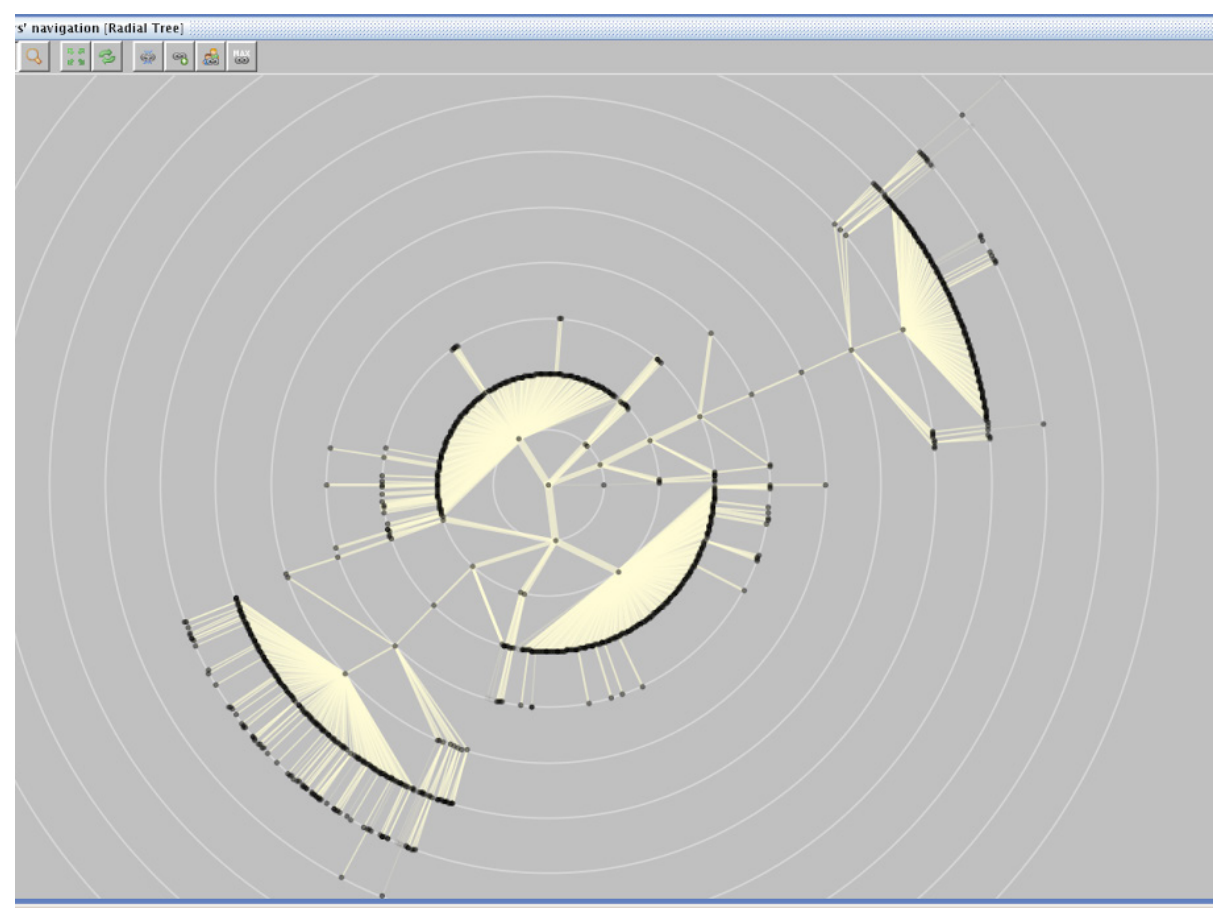

Figura 3 Exemplo de visualização na WET. Fonte: Dürsteler, Regidor e PascualCid, 2008

Das técnicas de representação levantadas na seção anterior, as mais apropriadas para representar a trajetória de aprendizagem são os grafos e as árvores, visto que ambas as técnicas representam elementos (i.e., páginas ou objetos de aprendizagem) e seus relacionamentos (i.e., a ordem de acesso aos elementos). Nos exemplos mostrados acima, Fortenbacher et al. (2013) adotaram técnicas de representação em rede (i.e., grafo) e em árvore (i.e., treemaps), enquanto Pascual-Cid (2008) representou a trajetória através de uma rede (i.e., grafo).

Segundo Stones e Sobol (2002) a visualização da trajetória do usuário é desafiadora por três motivos principais, são eles:

- Visitas repetidas a nós devem ser claramente identificáveis

- A sequência de visitação aos nós deve ser identificável

- A direção em que os nós são visitados deve ser visível

Desta forma, a implementação da visualização das trajetórias de aprendizagem dos alunos requer a identificação e análise dos dados obtidos pelos AVAs, bem como uma estruturação dos ambientes para que permitam identificar a sequência e a direção das páginas e objetos de aprendizagem visitados. Além disso, o estudo das técnicas de interação mais apropriadas a cada ambiente é necessário para entender as necessidades dos professores e/ou alunos ao visualizarem e interagirem com tais informações. Na próxima seção é apresentada a ferramenta de visualização da informação implementada em um ambiente e-learning. 


\section{Ferramenta de visualização}

Nesta seção descrevemos uma ferramenta de Visualização da Informação que permite a análise do comportamento dos alunos em um ambiente e-learning, bem como todas as etapas envolvidas no seu desenvolvimento. A primeira etapa do desenvolvimento desta ferramenta foi a análise do ambiente e-learning AdaptWeb ${ }^{\circledR}$, de forma a identificar quais áreas ajudam a entender o comportamento dos alunos. A segunda etapa consiste na coleta e análise de dados. Por fim, os resultados da análise de dados são transformados em representações gráficas de modo que o professor possa realizar as análises de seu interesse de forma rápida e intuitiva.

\subsection{Ambiente e-learning}

O AdaptWeb ${ }^{\circledR}$ (Ambiente de Ensino-Aprendizagem Adaptativo na Web) é um ambiente e-learning adaptativo, i.e., adapta o conteúdo, a apresentação e a navegação de uma disciplina de acordo com o perfil do aluno (e.g., conhecimento sobre um determinado conceito, preferências de navegação e histórico). Este ambiente e-learning foi escolhido por ser um ambiente open source (i.e., gratuito e que pode ser modificado/personalizado) e ser atualmente utilizado em nossa universidade. Este ambiente está disponível em <http://adaptweb. sourceforge.net/>.

Além de ser adaptativo, este ambiente permite oferecer uma disciplina em mais de um curso, e.g., a disciplina de Cálculo pode ser oferecida nos cursos de Ciência da Computação, Engenharia Elétrica e Engenharia Civil com o conteúdo devidamente adaptado para cada curso. No AdaptWeb ${ }^{\circledR}$ o aluno pode acessar três seções:

- Ambiente de Aula: Onde o aluno vê o conteúdo da disciplina e possui acesso a conceitos, exemplos, exercícios e materiais complementares. Ao acessar esta seção, o aluno pode optar pelo modo Tutorial (considera os pré-requisitos definidos pelo professor, determinando a navegação do aluno pelo conteúdo didático) ou pelo modo Livre (não considera os requisitos).

- Fórum de Discussão: Permite que os alunos interajam entre si criando discussões (tópicos) sobre um determinado assunto de seu interesse ou respondendo tópicos já criados, assim podem tanto ajudar quanto obter ajuda.

- Mural de Recados: O aluno pode ver mensagens de seu curso/ disciplina dos últimos 30 dias. Estas mensagens podem ser públicas ou privadas; e de outros alunos ou de seu professor. O aluno também pode enviar mensagens para os outros alunos e para seu professor.

Cada uma destas três seções é parte de uma disciplina de um determinado curso, e.g., na disciplina de Cálculo, um aluno do curso 
de Ciência da Computação vê, nas três seções, apenas o conteúdo disponível para alunos de Ciência da Computação; e um aluno de Engenharia Elétrica vê apenas o conteúdo disponível para os alunos da Engenharia Elétrica.

\subsection{Coleta e análise de dados}

Para coletar dados de interação dos alunos com o ambiente, foi implementada uma ferramenta web analytics no ambiente e-learning escolhido, de forma que os dados são coletados nas três seções que o aluno pode acessar (i.e., Ambiente de Aula, Fórum de Discussão e Mural de Recados). A partir destes dados, algumas métricas relacionadas ao conteúdo, à navegação e ao cumprimento de metas dentro do ambiente - são calculadas. Estas métricas foram divididas em quatro categorias, de modo a facilitar a compreensão do professor, e são:

- Uso geral: Métricas não relacionadas às três seções principais, i.e., Total de Visitas por Aluno, Tempo Médio de Acesso dos Alunos, Frequência de Acesso, Total de Acessos a Cada Seção, Sistema Operacional, Navegadores e Resoluções de Tela;

- Ambiente de Aula: Métricas relacionadas ao Ambiente de Aula, i.e., Modo de Navegação, Total de Usos do Sistema de Busca, Palavras-chave Pesquisadas, Total de Acessos aos Conceitos, Total de Acessos aos Exercícios, Total de Acessos aos Exemplos e Total de Acessos aos Materiais Complementares;

- Fórum de Discussão: Métricas relacionadas a utilização do Fórum de Discussão, i.e., Total de Acessos aos Tópicos, Total de Tópicos Criados e Total de Respostas;

- Mural de Recados: Métricas para analisar a troca de recados dos alunos, i.e., Total de Recados Enviados, Tipos de Recados Enviados (para os outros alunos ou para o professor) e Total de Visualizações.

\subsection{Representação visual}

Com base no ciclo proposto por Card, Mackinlay e Shneiderman (1999), os dados referentes a cada uma das métricas calculadas pela ferramenta web analytics passam por um processo de transformação. Utilizando como exemplo a métrica Total de Visitas por Aluno, os dados calculados pela ferramenta web analytics são transformados em uma estrutura de dados similar a uma tabela, onde cada aluno possui um valor que representa o seu número de acessos. A partir desta estrutura, cada aluno se torna uma barra no gráfico de barras, cujo tamanho representa o total de visitas. Ao exibir o gráfico resultante para o professor ele pode alterar algumas propriedades 
do gráfico ou dos dados para melhorar a sua compreensão sobre o conjunto de dados em análise. A seguir, descrevemos a estrutura da ferramenta desenvolvida, bem como as técnicas de representação e interação aplicadas.

Conforme a Figura 4, a ferramenta de visualização possui quatro áreas principais: Filtros (Figura 4.1), Seleção de Métricas (Figura 4.2), Gráfico (Figura 4.3) e Tabela de Detalhes (Figura 4.4). O filtro (uma técnica de interação que permite que o conjunto de dados em análise seja alterado) permite que o professor selecione os dados de acordo com o curso, a disciplina e o período (uma data inicial e uma data final) que deseja analisar. A seleção de métricas mostra as métricas disponíveis e o professor pode escolher qual irá analisar. A maioria delas é analisada de forma individual, no entanto, algumas destas métricas podem ser analisadas em conjunto. É caso das métricas Total de Acessos aos Conceitos, Total de Acessos aos Exercícios, Total de Acessos aos Exemplos e Total de Acessos aos Materiais (da categoria Ambiente de Aula) que, quando analisadas em conjunto, permitem verificar qual(is) das categorias obteve mais acessos. Outras métricas que podem ser analisadas em conjunto são Total de Acessos aos Tópicos e Total de Respostas (da categoria Fórum de Discussão), permitindo verificar a relação entre acessos e respostas.

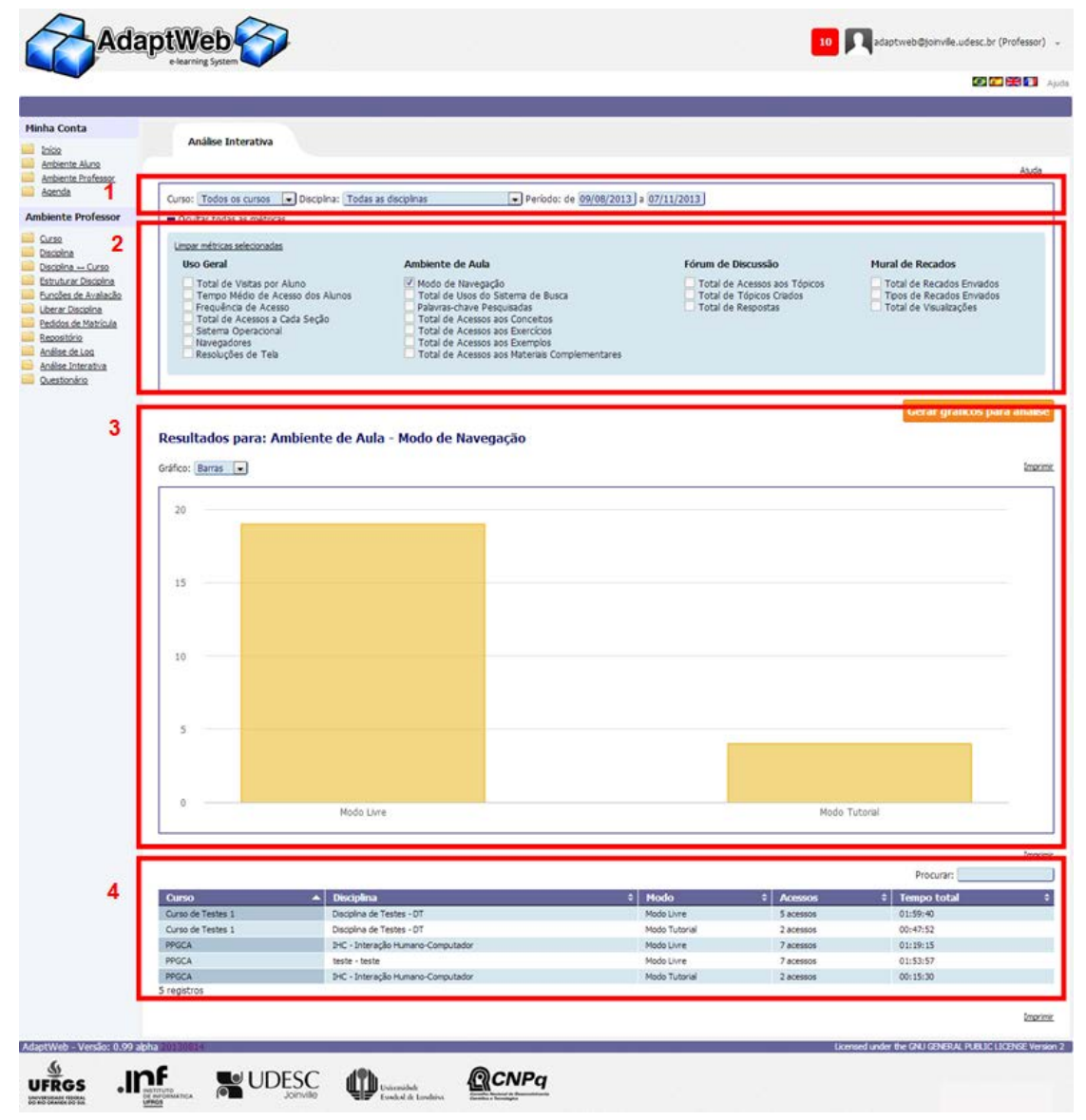

Figura 4 Ferramenta de análise da interação dos alunos com o ambiente Fonte: adaptado de Moissa e Gasparini (2014). 
Após o professor selecionar os dados e a(s) métrica(s) que deseja analisar, os resultados são exibidos em gráficos de barras, gráfico de linhas ou gráfico de setores (escolhidos pois são técnicas de representação ortogonais adequadas para as métricas implementadas, já que possuem poucas variáveis). Algumas técnicas de interação aplicadas aos gráficos são a possibilidade de agrupar a métrica Frequência de Acessos por dia, semana ou mês; escolher se o gráfico de setores terá rótulos ou legendas; obter detalhes sobre um item do gráfico (ponto, barra ou setor) ao descansar o ponteiro do mouse sobre ele. Há também a possibilidade de analisar informações mais detalhadas através da tabela abaixo do gráfico. Para auxiliar, o professor pode filtrá-los por uma palavra-chave e ordenar as linhas de acordo com os valores de uma das colunas.

\subsection{Perspectivas futuras}

No trabalho de Carvalho e Gasparini (2013) uma pesquisa com 22 professores universitários de cinco estados brasileiros (Rio Grande do Sul (UFRGS, PUCRS, UFPel, FURG e UCPel), Santa Catarina (UDESC, Instituto Federal Catarinense), Paraná (UFPR, UTFPR e UNIOSTE), Mato Grosso (UFMT), São Paulo (USP), Rio de Janeiro (UFF) e Pará (UFPA)) foi realizada através de um questionário para identificar as necessidades e objetivos do professor em ambientes e-learning.

Os principais recursos destacados foram incorporados no ambiente AdaptWeb ${ }^{\circledR}$ (e.g. visualizações e estatísticas sobre uma disciplina ou aluno tais como frequência do aluno, realização das tarefas, etc.). Um recurso considerado importante por professores foi a visualização do Caminho Percorrido pelo Aluno, ou seja, sua trajetória de aprendizagem. A visualização da trajetória de aprendizagem do aluno ainda não foi implementada nesta ferramenta, porém, a ferramenta web analytics do AdaptWeb ${ }^{\circledR}$ já coleta os dados necessários para realizar esta análise.

Desta forma, como trabalho futuro, será implementada a trajetória de aprendizagem do aluno em cada disciplina do ambiente. Essa será dinâmica e interativa, de modo a facilitar a compreensão por parte do professor.

\section{Considerações finais}

Devido ao grande volume de dados coletados por diversos tipos de sistemas, as técnicas de visualização da informação estão sendo utilizadas com diversos objetivos, e auxiliam a um melhor entendimento visual da análise dos dados. Dentre estes objetivos encontra-se a utilização das técnicas para analisar o comportamento de usuários frente a sistemas e, inclusive, em ambientes e-learning. 
Este artigo apresenta o conceito de visualização da informação e suas técnicas de representação e interação. Além disso, apresenta uma ferramenta de visualização da informação do ambiente AdaptWeb ${ }^{\circledR}$. Os dados coletados e as métricas calculadas pela ferramenta objetivam analisar o comportamento dos usuários frente ao sistema. Desta forma, os professores têm suporte para entenderem melhor o comportamento de seus alunos e identificarem problemas de aprendizagem, fazendo com que possam interferirem antes de um possível problema acontecer.

Além das métricas já implementadas no ambiente, tais como análise de frequência, tópicos vistos, porcentagem de exercícios concluídos, etc., estamos interessados em implementar visualmente a trajetória de aprendizagem do aluno.

Atualmente, estamos em um processo de estudo do estado da arte na área de visualização da trajetória de aprendizagem. Esse estudo está sendo realizado com base em algumas questões de pesquisa, tais como:

- A trajetória de aprendizagem do aluno é verificada em ambientes e-learning?

- Como os ambientes capturam/detectam essa informação?

- Que análises são realizadas?

- Quais técnicas são utilizadas?

- Como é a visualização do caminho encontrado?

- Quais as consequências no processo de ensino-aprendizagem?

Após a análise dessas questões, esperamos definir como será realizada a visualização das trajetórias com base no que está sendo realizado por outros autores, acrescentando-as às outras visualizações já presentes na ferramenta.

\section{Agradecimento}

Este trabalho é parcialmente apoiado pela FAPESC, CAPES e CNPq. Agradecemos a CAPES e CNPq pelas bolsas de estudo concedidas. 


\section{Referências}

CAMPO, M.; OROSCO, R. \& TEYSEYRE, A. 1997. Automatic Abstraction Management in Information Visualization Systems. Proceedings of the International Visualization Conference: 50-56.

CARD, S. K.; MACKINLAY, J. D. \& SHNEIDERMAN, B. 1999. Readings in Information Visualization: using vision to think. Morgan Kauffman.

CARVAlho, L. S. DE \& GASPARINI, I. 2013. Compreensão dos objetivos do professor nos ambientes e-learning para análise de métricas web analytics. Conferência Ibero-Americana WWW/Internet (CIAWI), Porto Alegre.

CHI, E. H. \& RIEDL, J. T. 1998. An operator Interaction Framework for Visualization Spreadsheets. Proceedings of IEEE Information Visualization Symposium: 63-70.

DÜRSTELER, J. C.; REgIDOR, S. M. \& PASCUAL-CID, v. 2008. How to use WET. Inf@Vis! Disponível em <http://www.infovis.net/printMag. php?num=194\&lang=2>. Acesso em 20 dez 2014.

FREITAS, C. M. D. S.; CHUBACHI, O. M.; LUZZARDI, P. R. G. \& CAVA, R. A. 2001. Introdução à Visualização de Informações. Revista de Informática Teórica e Aplicada, v.8, n.2: 143-158.

FORTENBACHER, A.; BEUSTER, L.; ELKINA, M.; KAPPE, L.; MERCERON, A.; PURSIAN, A.; SCHWARZRock, S. \& WENZLAFF, B. 2013. LeMo: a Learning Analytics Application Focussing on User Path Analysis and Interactive Visualization. The 7th IEEE International Conference on Intelligent Data Acquisition and Advanced Computing Systems: Technology and Applications.

GHERSON, N.; EICK, S. G. \& CARD, S. 1998. Information Visualization. Interactions, New York, NY, USA, v. 5, n. 2: 9-15.

GRAHAM, M. \& KENNEDY, J. 2010. A survey of multiple tree visualisation. Information Visualization. v. 9, n. 4: 235-252.

HABER, R. B. \& MCNABB, D. A. 1990. Visualization Idioms: a conceptual model for scientific visualization systems. Visualization in Scientific Computing: 74-93.

MOISSA, B. \& GASPARINI, I. 2014. Técnicas de visualização da informação para analisar o comportamento de alunos em um ambiente E-Learning. Anais do Computer on the Beach: 263-272.

PALAZZO, J.; VALDENI, J.; KRUG, L.; MARILZA, A.; GASPARINI, I.; FERNÁNDEZ, A. \& DÍAZ, A. 2014. Adaptatividade geocultural em ambientes virtuais de aprendizagem. RIED v. 17, n.1: 83-109.

PASCUAL-CID, V. 2008. An information visualisation system for the understanding of web data. IEEE Symposium on Visual Analytics Science and Technology: 183-184.

SCHOONENBOOM, J., LEVENE, M., HELLER, J., KEENOY, K. \& TURCSANYI-SZABO, M. 2007. Trails in Education: Technologies that Support Navigational Learning. Sense Publishers.

STASKO, J. 2008. Visualization for Information Exploration and Analysis. IEEE Symposium on Visual Languages and Human-Centric Computing: 7-8.

STONES, C. \& SOBOL, S. 2002. DMASC: A Tool For Visualizing User Paths Through A Web Site. IEEE Database and Expert Systems Applications. 
TARAWNEH, R. M.; KELler, P. \& EBERT, A. 2011. A General Introduction To Graph Visualization Techniques. Proceedings of IRTG 1131 Workshop 2011, OASICS, Vol. 27, VLUDS 2011, June 10-11, Kaiserslautern, Germany.

VAZ, F. R. \& CARVALHO, C. L. 2004. Visualização de Informações. Relatório técnico Universidade Federal de Goiás.

YI, J. S.; KANG, Y.; STASKO, J. T. \& JACKO, J. A. 2007. Toward a Deeper Understanding of the Role of Interaction in Information Visualization. IEEE Transactions on Visualization and Computer Graphics: 1224-1231.

\section{Sobre os autores}

\section{Barbara Moissa}

Bacharel em Ciência da Computação pela Universidade do Estado de Santa Catarina (UDESC) e mestranda no Programa de PósGraduação em Computação Aplicada também na UDESC. Pesquisa na área de Visualização da Informação, Learning Analytics e Interação Humano-Computador. <barbara.moissa@gmail.com>

\section{Eduardo José Borba}

Graduando no Bacharelado em Ciência da Computação pela Universidade do Estado de Santa Catarina (UDESC). Bolsista de iniciação científica nas áreas de Visualização da Informação e Learning Analytics.

<eduardojoseborba@gmail.com>

\section{Avanilde Kemczinski}

Doutora em Engenharia de Produção e Sistemas pela Universidade Federal de Santa Catarina (UFSC), professora na Universidade do Estado de Santa Catarina (UDESC) e líder do Grupo de Pesquisa em Informática na Educação (GPIE) pelo CNPQ/UDESC. Tem interesse nas áreas correlatas a Informática na Educação e Interação Humano-Computador. <avanilde.kemczinski@udesc.br>

\section{Isabela Gasparini}

Doutora em Ciência da Computação pela UFRGS (Universidade Federal do Rio Grande do Sul). Professora da UDESC. Atua na área de Interação Humano-Computador. Seus trabalhos incluem a área de visualização da informação e usabilidade.

<isabela.gasparini@udesc.br>

Artigo recebido em 16 out. 2014, aprovado em 11 dez. 2014. 
Moissa, B., Borba, E. J., Kemczinski, A. \& Gasparini, I. | Uma ferramenta de Visualização da Informação para analisar o comportamento do aluno em um ambiente e-learning e sua trajetória de aprendizagem 